\title{
THE ONTOGENY OF CIRCADIAN RHYTHMS OF SEVERAL PHYSIOLOGICAL PARAMETERS IN MALE WISTAR RATS
}

Article history:

Submitted 3 June 2019

Accepted 20 July 2019

\section{David Areshidze", Lyudmila Makartseva, Sergey Kucher, Angelica Gritsyunayte, Kristina Sakhnova, Vladimir Kaplan, Egor Shmigelskiy, Maria Kozlova}

\author{
Laboratory of experimental biology and biotechnology. \\ Moscow State Regional University, Moscow, Russia
}

*Corresponding Author: notbio@mgou.ru

ABSTRACT - The rhythmicity of biological processes is one of the fundamental properties of living matter. Rhythmic effects from the external environment are the main stimulators of the biorhythms of the organism, which ensure their formation in the early stages of ontogenesis and determine the level of their intensity throughout all subsequent life. Among the environmental factors the leading is photoperiodicity. Exposure to light at night has become an essential part of the modern lifestyle and it is accompanied by many serious behavioral and health disorders, including cardiovascular diseases and cancer. To make a more complete picture of the patterns of temporary organization in mammals it occurs currently important to study the characteristics of the daily dynamics of some physiological parameters of male Wistar rats in ontogenesis.

The study was conducted on Wistar male rats at the age of 3, 6 and 12 months. Each age group was divided into 2 subgroups, comprising 40 animals in each of them. The rats of first subgroup of each studied age were housed under a fixed illumination, animals of second subgroup of each of studied ages were investigated under the same experimental conditions except for the light regime, representing constant light. The daily rhythmic of blood biochemical parameters and rhythmic of rectal temperature were studied. For the analysis of characteristics of circadian rhythm of the studied substances the cosinor-analysis carried out by means of the Cosinor Ellipse 2006-1.1 program was used. A study of the characteristics of the daily rhythm of a number of parameters of Wistar albino rat males at the age of 3, 6 and 12 months at a fixed light regime and under constant light conditions was conducted. Keeping of rats in conditions of constant illumination leads to significant violations in the structure of the chronoarchitecture of the organism. This is manifested in a change in the nature of the daily dynamics of rectal temperature, a significant reorganization of the circadian rhythm of glucose, a disturbance of the circadian rhythm of the total protein and albumin, which together indicates the development of desynchronosis and stress of adaptation mechanisms. It was established that the stability of the circadian system of the rat organism manifests different degrees of maturity in the studied periods of ontogenesis, and also reacts differently to changes in the light regime. The animals at the age of 6 months are the most resistant to the effects of constant lighting.

KEYW ORDS - circadian rhythm, ontogenesis, temperature, albumin, glucose, total protein

\section{NTRODUCTION}

The rhythmicity of biological processes is one of the fundamental properties of living matter. Rhythmic effects from the external environment are the main stimulators of the biorhythms of the organism, which ensure their formation in the early stages of ontogenesis and determine the level of their intensity throughout all subsequent life. Thus, the exposures from the external environment are the factor that set the biological clock of organism and determines the specifics of their rate. For living organisms of different levels of organization the presence of evolutionarily determined endogenous circadian rhythm is characteristic. Each mammalian cell is a potential oscillator, as it contains the necessary elements that determine the molecular genetic mechanisms of circadian biological clock (BC), which can be activated under certain conditions. Balsalobre (2002); Kim et al. (2018).

The chronoperiodic system, being present at all levels of the organization of a living organism, generates oscillations of its own activity with frequencies that are close to the frequencies of the main external geophysical cycles (daily, monthly, annual), and is capable of capturing external timers (Zeitgeber) and thus synchronizing its own activity with external rhythmic changes. Carter et al. (2016); Dibner, C., Schibler, U. (2015); Erkekoglu and Baydar (2012); Halberg (2005).

Among the environmental factors the leading is photoperiodicity. Photoperiodicity (the duration of daily and/or seasonal illumination) is the main pacemaker for mammals (time-setting cue or external synchronizing factor) Wehr (2001); Foster (2008); Boyce (2014).

It is known that information about the illuminance enters the organism through the retina. Currently, it has been shown that, in addition to retinal receptors, ganglion and bipolar cells also take part in the perception of the photoperiod. In these cells, the action of the light waves of the blue spectrum leads to the induction of cry genes that produce cryptochrome proteins from the group of flavoproteins. The accumulation of such proteins 
can provide the primary perception of photoperiodic information in living organisms. Tang et al. (2010); Schmidt et al. (2011); Nasir-Ahmad et al. (2019).

Then the photoperiodic information goes along the optic nerve, which contains the retinohypothalamic tract, reaching the suprachiasmatic nuclei. There is also an indirect path through which light information reaches the suprachiasmatic nuclei - the geniculohypothalamic pathway. This path is a projection from parts of the lateral geniculate nuclei that receive retinal information, as well as from the ventral parts of these nuclei. Photoperiodic information comes to the geniculate nuclei by direct projections from the retina which comprise into the main part of the optic nerve. It is assumed that the geniculohypothalamic tract contributes to suprachiasmatic nuclei's obtaining of modulating signals from other sensory and motor systems of the organism. Rusak (1992); Harrington (1997); Ding (2018).

The key role of the suprachiasmatic nuclei of the hypothalamus in the chronoperiodic system of mammals is unquestionable.

Suprachiasmatic nuclei have all the properties of oscillators. Hastings et al. (2019):

- they are endogenous pacemakers, i.e. oscillators with intrinsic circadian frequency of neuronal activity;

- they are capable to capture the rhythm of an external synchronizer - a photoperiod - through direct and indirect communication with the retina of the eye and can synchronize their own rhythms with an external photoperiod;

- they are the main synchronizers for many endogenous innate rhythms of mammals.

SCN fully or partially synchronize locomotor activity, food and water consumption, reproductive behavior, temperature regulation, sleep-wake cycle, daily rhythm of heart contractions. At the same time, suprachiasmatic nuclei implement a synchronizing effect on the circadian rhythm of the endocrine system. Martino and Young (2015). By means of various connections, the SCN can manage the temporal organization of homeostatic systems, synchronizing their rhythms. In addition, the SCN can regulate circadian activity by affecting the centers of the autonomic nervous system through the caudal parts of their efferents. Chiesa et al. (2015); Moller et al. (2009) Sympathetic fibers, in turn, provide innervation to the pineal gland, another important link in the mammalian photoperiodic system. lovino et al. (1986).

In addition to the adrenergic innervation, the pineal body also receives a variety of non-adrenergic nerve fibers of both peripheral and central origin, but their effect remains little studied.
The formation of melatonin in mammals almost exactly corresponds to the activity of postganglionic sympathetic neurons, which depends primarily on the photoperiod phase; all cells of the organism can react to the state of the external zeitgeber by the level of melatonin in the circulating blood. Therefore, melatonin is sometimes referred to as an internal zeitgeber, and the pineal body is called a neuroendocrine transducer, which converts information on the duration of a photoperiod, encoded in nerve impulses, into a humoral response in the form of circulating melatonin. Melatonin is produced in the dark, and its main function is chronoregulation, although melatonin can simultaneously perform various other functions Robert et al. (2015); Touitou et al. (2006).

The circannual part of the photoperiodic system of the brain has been studied significantly less. It has been established that in many organisms the circadian rhythms are involved in the control of circannual changes, since the former take part in the mechanisms of photoperiodic time measurement, perceiving the rhythms of external pacemakers.

The circadian (context-independent) system can only inform the organism about the difference between the light and dark periods of the day or between low and high melatonin levels, but not about the seasonal reduction or lengthening of the photoperiod Yurt et al. (2018).

It is supposed that the suprachiasmatic nuclei can not only be a circadian oscillator (pacemaker), but also take part in seasonal photoperiodic timekeeping, that is, conduct a seasonal estimate of time, be a photoperiodic counter of time Schwartz et al. (2001).

It is also possible to consider the involvement of the nuclei of the anterior hypothalamus (anterior, suprachiasmatic, preoptic and others) in the transmission of information from the circadian system to the photoperiodic comparator. And the disappearance of photoperiodic reactions due to the disruption of the activity of the anterior hypothalamic nuclei is associated with blocking the transmission of information from the circadian system to a such comparator.

The septohippocampal system is proposed as a such comparator. The assumption is due to the facts that:

- one of the neuroanatomical memory substrates is the hippocampus;

- the hippocampus together with the septal complex forms a joint morpho-functional septohippocampal system, which can function both in the direction of the "medial septal nucleus - hippocampus - the lateral septal nucleus - medial septal nucleus", and, possibly, in the opposite direction;

- the septohippocampal system, due to its diverse connections, has an ideal arrangement for the comparator; 
- taking into account the morphofunctional features of the structure, the septo-hippocampal system is already considered a comparator, but with a different function - acting as a generator of consciousness and attention;

- there is evidence of involvement of the hippocampus and the entire hippocampal formation in the occurrence of photoperiodic reactions. In addition, it has been demonstrated that the adaptation effects of the pineal body are realized through the changes in the functional state of the hippocampus. Arushanyan (2001); Serrano et al. (2006).

Currently a fairly large number of people in the world are exposed to light pollution (in other words, night lighting). Such an impact may be due to the profession, or due to habits and lifestyle. Exposure to light at night has become an essential part of the modern lifestyle and is accompanied by many serious behavioral and health disorders, including cardiovascular diseases and cancer $\mathrm{Ha}$ (2005); Jasser et al. (2006); Knutsson (2003). Inhibition of the function of the epiphysis (pineal gland) in constant light contributes a carcinogenesis, while an absence of light suppresses carcinogenesis.

According to the hypothesis of circadian disruption, the effect of light at night violates the endogenous circadian rhythm, suppresses the melatonin secretion by epiphysis, which leads to a decrease in its concentration in the blood Wang et al. (2014). Disturbance of CR during shift working leads to increased risk of cardiovascular diseases, metabolic syndrome, type II diabetes. Fonken et al. (2010). Animal studies have shown that replacing of total darkness with dim night lighting causes metabolic disturbances and obesity Vinogradova et al. (2013); Gubin et al. (1997). Thus, the violation of the CR negatively affects eating behavior and metabolism.

The temporal organization of biological processes in mammals undergoes complex ontogenetic transformations, which reach their maximum during aging, and in general can be characterized as age-related desynchronosis, which plays a significant role in the development of aging processes and the development of diseases associated with it. In the process of aging, there is a gradual loss of synchronism of factors involved in the modulation of the amplitude-phase characteristics of phenotypic rhythms (physiological, biochemical processes, behavioral reactions, etc.). These factors can be molecular-genetic, tissue-organ and systemic. Phenotypic manifestations of desynchronosis are formed according to the principles of pleiotropy. It is assumed that the general manifesta- tion of age-related desynchronosis on the part of the rhythm architectonics is extracircadian dissemination (ECD) - a violation, and most often - a decrease in the amplitude of the circadian rhythm (CR) with the background of the loss of its phase stability and an increase in oscillations in adjacent frequency ranges Gubin et al. (1999); Gubin et al. (2001); Gubin et al. (2006).

To make a more complete picture of the patterns of temporary organization in mammals it occurs currently important to study the characteristics of the daily dynamics of some physiological parameters of male Wistar rats in ontogenesis.

\section{MATHERIALS AND METHODS}

The study was conducted on Wistar male rats at the age of 3, 6 and 12 months. Animals were taken from the Stolbovaya nursery (the Stolbovaya affiliate of the Federal State Budgetary Institution of Science "Scientific Center for Biomedical Technologies of the Federal Medical and Biological Agency"). Each age group was divided into 2 subgroups, comprising 40 animals in each of them.

Treatment design

Animals of each of the studied ages were initially kept under a fixed light regime.

During the whole experiment, the rats of first subgroup of each studied ages were housed under a fixed illumination, L:D 10:14 ( \pm 180 lux, respectively; 8:00 AM lights on) in a temperature-controlled environment with ad libitum access to tap water and food (rat chow).

Animals of second subgroup of each of studied ages were investigated under the same experimental conditions except for the light regime, representing constant light ( $\mathrm{LL} \pm 180$ lux). Both the first and second subgroups of animals were kept at the specified light regime for 2 weeks.

After two weeks, euthanasia of the animals in the carbon chamber was performed at 0900 hours, 1500 hours, 2100 hours and 0300 hours, blood was collected for biochemical studies.

All animal experiments were performed in according to the compliance with EC Directive 86/609/EEC and with the Russian law regulating experiments on animals.

Biochemical studies

In the blood plasma the levels of alanine aminotransferase (ALT), aspartate aminotransferase (AST), total bilirubin, cholesterol, triglycerides, total protein, glucose, albumin and uric acid were deter- 
mined using the StatFax-3300 (USA) analyzer and corresponding Spinreact kits (Spain).

\section{Rectal temperature studies}

For measurement of rectal temperature, the BIOTK8851 thermometer (Bioseb, USA) was used.

\section{Statistical Analysis}

The obtained data, analyzed using Graph Pad Prism 6.0, were expressed as Mean \pm SD. The statistical difference was determined using Student t-tests. A p value of $<0.05$ was considered statistically significant.

For the analysis of characteristics of circadian rhythm of the studied substances the cosinor-analysis carried out by means of the Cosinor Ellipse 2006-1.1 program was used. The cosinor analysis allows to analyze the chronobiological data, to reveal the rhythm of various functional parameters, and also to calculate the parameters of the established rhythms Cornelissen (2014). The presence of a reliable circadian rhythm, and also its acrophase and amplitude were determined. Acrophase is the measure of peak time of the total rhythmic variability in a 24-hour period. Amplitude corresponds to a half of the total rhythmic variability in a cycle. The acrophase is expressed in hours; amplitude values are expressed with the same units as the documented variables.

\section{RESULTS}

The daily dynamics of rectal temperature in rat's groups of different ages at a fixed light regime and in conditions of constant lighting.

The study of rectal temperature of animals at the age of 3 months have found a circadian rhythm with acrophase at 0132 hours, low amplitude $-0.18^{\circ} \mathrm{C}-$ and $35.68^{\circ} \mathrm{C}$ mesor. Transition to a constant lighting leads to the destruction of the rectal temperature rhythm (Fig. 1).

In rat's group aged 6 months we observed a circadian rhythm of temperature, characterized by acrophase, falling at 1615 hours, with a mesor of $35.43^{\circ} \mathrm{C}$ and an amplitude of $0.68^{\circ} \mathrm{C}$. At the same time, the transition to keeping animals in conditions of constant illumination leads to breakdown of circadian rhythm.

In rat's group of the third studied age, both at fixed light regime and under constant illumination the circadian rhythm of temperature is marked. In the first case it is characterized with acrophase at 0458 hours, and at constant light the acrophase shifts to 1308 hours. At the same time, the amplitude of the rhythm increases from $0.62^{\circ} \mathrm{C}$ to $0.77^{\circ} \mathrm{C}$, with almost unchanged mesore $-34.92^{\circ} \mathrm{C}$ and $34.33^{\circ} \mathrm{C}$, respectively.

\section{The daily dynamics of plasma glucose level in rat's groups of different age at a fixed light regime and in conditions of constant lighting.}

Analysis of the daily dynamics of blood glucose at a fixed light regime revealed the presence of a reliable circadian rhythm in rats of all studied ages (Fig. 2). It is noteworthy that consideration of its changes shows a tendency towards directional displacement of its acrophase (1600 hours in group of 3 months age, 1157 in 6-months-old group and 0837 hours at 12 months). The rhythm amplitude is $0.49 \mathrm{~g} / \mathrm{l}$ at 3-months-old group, $0.9 \mathrm{~g} / \mathrm{l}$ at 6 months and $0.76 \mathrm{~g} / \mathrm{l}$ at 12 months. The rhythm mesor was $7.58 \mathrm{~g} / \mathrm{l}$ in 3-months-old rats, $8.26 \mathrm{~g} / \mathrm{l}$ at 6 months and $6.97 \mathrm{~g} / \mathrm{l}$ for 12 months.

Under the constant light conditions the circadian rhythmicity of plasma glucose level was detected at 3-and 6-months-old groups. Acrophase of rhythms shifted at 1920 and 2030 accordingly, the rhythm amplitude at 3-months-old rats was $1.15 \mathrm{~g} / \mathrm{l}$ and $1.57 \mathrm{~g} / \mathrm{l}$ at 6-months-old. Mesor is respectively equal to $6.87 \mathrm{~g} / 1$ and $8.53 \mathrm{~g} / \mathrm{l}$.

The daily dynamics of total plasma protein in rat's groups of different age at a fixed light regime and in conditions of constant lighting.

At a fixed light regime in animal's group aged 3 months no reliable circadian rhythm of total plasma protein was detected. At 6 months the rhythm is characterized by acrophase of 0230 hours, with an amplitude of $7.52 \mathrm{~g} / \mathrm{l}$ and mesor of $73.44 \mathrm{~g} / \mathrm{l}$. At 12 months, the acrophase of rhythm is noted at 0055 , the amplitude decreases, amounting $6.48 \mathrm{~g} / \mathrm{l}$ with a mesor of $60.23 \mathrm{~g} / \mathrm{l}$. In conditions of constant illumination in group of 3 months, the rhythm with acrophase at 2100 hours, an amplitude of $7.52 \mathrm{~g} / \mathrm{l}$ and mesor of $73.44 \mathrm{~g} / \mathrm{l}$ is observed. At 6 months in the same conditions the acrophase of rhythm is noted at 1242 , the amplitude sharply increases to $24.85 \mathrm{~g} / \mathrm{l}$ with a mesor of $60.23 \mathrm{~g} / \mathrm{l}$. In animals at the age of 12 months circadian rhythm is not detected (Fig. 3).

The daily dynamics of plasma albumin in rat's groups of different age at a fixed light regime and in conditions of constant lighting.

At a fixed light regime in rat's group aged 3 months acrophase rhythm falls on 0010 hours, the amplitude is $8.86 \mathrm{~g} / \mathrm{l}$, mesor $-21.13 \mathrm{~g} / \mathrm{l}$. At 6 months the rhythm acrophase shifts to 1500 , the amplitude is reduced to $2.95 \mathrm{~g} / \mathrm{l}$, the mesor is $8.46 \mathrm{~g} / \mathrm{l}$. At 12 months the acrophase of this rhythm falls at 0615 hours, the amplitude is $0.76 \mathrm{~g} / \mathrm{l}$, and the mesor is $4.95 \mathrm{~g} / \mathrm{l}$.

At constant light a reliable circadian rhythm is noticed at all ages. At the same time in 3 months it is characterized with acrophase at 1108 hours, an amplitude of $5.71 \mathrm{~g} / \mathrm{l}$ and a mesor of $11.48 \mathrm{~g} / \mathrm{l}$. At 6 months rhythm acrophase shifted to 1125 hours, the ampli- 
tude was $0.55 \mathrm{~g} / \mathrm{l}$, and mesor $-4.27 \mathrm{~g} / \mathrm{l}$. At 12 months, the acrophase is noted at 1845 hours, the amplitude is equal to $0.29 \mathrm{~g} / \mathrm{l}$, and the mesor is $3.14 \mathrm{~g} / \mathrm{l}$ (Fig. 4).

\section{DISCUSSION AND CONCLUSION}

Consideration of the results of changes in the daily dynamics of rectal temperature in ontogenesis suggests that at rat males of all investigated ages at a fixed light regime the reliable circadian rhythm is noted, while keeping animals in conditions of constant illumination leads to the establishment of reliable circadian rhythm only in animals aged 12 months. We can suggest that in the first two investigated ages (3 and 6 months) lack of circadian rhythm of temperature is associated with the violation of photoperiodicity and relative dysmaturity of functional systems maintaining homeostasis in animals of these groups, and detecting of circadian rhythm of rectal temperature at constant light at 12 months may due to the fact that during this period of ontogenesis under these conditions the change of light and darkness is not the lead pacemaker, but some kind of another factor is.

Information received by us is confirmed by a number of studies, according to which the optimal biorhythmological structure of body temperature of mammal starts to form in childhood and up to mature age persists in close boundaries (both on the mesor and on daily amplitude). In the future, the level of body temperature as a whole and of its circadian amplitude decreases. Especially this trend is expressed in old age. In general, since old age, the chronodesm of temperature homeostasis in humans changes abruptly. Its boundaries in the senior age groups are significantly lower down at the temperature scale. In our opinion, a decrease in the temperature level homeostasis and its diurnal rhythm is due to a decrease in energy potential of the cells of the organism. The deviation from the energy optimum of organism undoubtedly leads to a decrease in balance of biosystem as a whole, followed by an entropy growth and change of the most important indicator of the level of viability - the amplitude of the daily rhythm. Thus, the body temperature undergoes a daily rhythm synchronous in profile and amplitude to the structure of the daily rhythm of energy and plastic metabolism parameters established at the cellular and tissue levels.

In analysis of the circadian rhythm of glucose at a fixed light regime it is noticeable the presence of age drift of acrophase to the early morning hours. Changing of the lighting regime leads to a rerun of the circadian rhythm at the age of 3 and 6 months and its absence in 12 months. The level of total plasma protein has the circadian rhythm at fixed light regime only from the age of 6 months, while in conditions of constant illumination the daily rhythm of this parameter is noted at 3 and 6 months and collapses at 12 months. The circadian rhythm of albumin is noted as at a fixed light, and at constant illumination in groups of all studied ages, but in both lighting regimes there is a significant decrease in albumin rhythm amplitudes. Thus, studies have shown that keeping of rats in constant light conditions leads to significant violations in the structure of the chronoarchitecture of the organism. It manifests in a change in the nature of the daily dynamics of rectal temperature, significant alteration of the circadian rhythm of glucose, violation of the circadian rhythm of total protein and albumin, which is cumulative talking about the development of desynchronosis and stress of mechanisms of adaptation. The most resistant to constant lighting are animals at age of 6 months.

A study confirms the fact that the violation of such an important factor as normal photoperiodism causes stress of mechanisms of adaptation of mammals and serves the cause of the formation of a general adverse background, conducting to a development of pathologies and pathological conditions.

\section{Acknowledgements}

Financial support for this study was carried out by Moscow State Regional University.

The authors declare that there is no conflict of interests regarding the publication of this paper.

\section{REFERENCES}

Balsalobre, A. 2002. CLOCK genes in mammalian peripheral tissues. Cell Tissue Res., 309:199-193. doi: $10.1007 /$ s00441-002-0585-0

Kim P, OSTer H, Lehnert H, Schmid SM, Salamat N, BARCLAY JL. 2018. Coupling the circadian clock to homeostasis: the role of period in timing physiology. Endocrine reviews. 40(1):95-66. doi.org/10.1210/ er.2018-00049

Carter, S. J., Durrington, H. J., GibBs, J. E., BlaikLEY, J., Loudon, A. S., RaY, D. W., SABroe, I. 2016. A matter of time: study of circadian clocks and their role in inflammation. Journal of leukocyte biology, 99(4), 549-560.

Dibner, C., SChibler, U. 2015. Circadian timing of metabolism in animal models and humans. Journal of internal medicine, 277(5), 513-527. https://doi. org $/ 10.1111 /$ joim. 12347

Erkekoglu, P., Baydar, T. 2012. Chronopharmacodynamics of drugs in toxicological aspects: A short review for clinical pharmacists and pharmacy practitioners. Journal of research in pharmacy practice, 1(2), 41. doi: $10.4103 / 2279-042 X .108369$ 


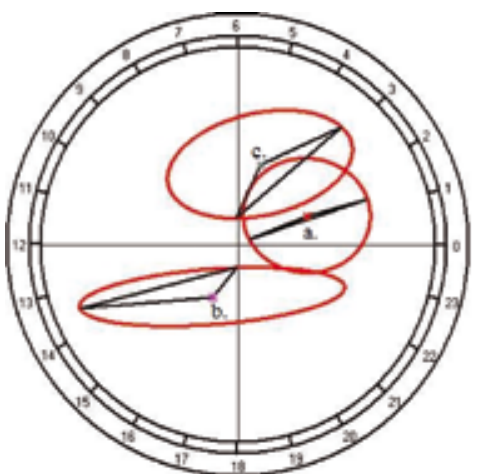

a

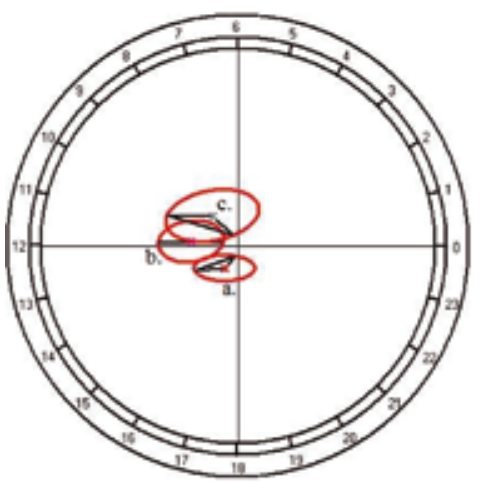

a

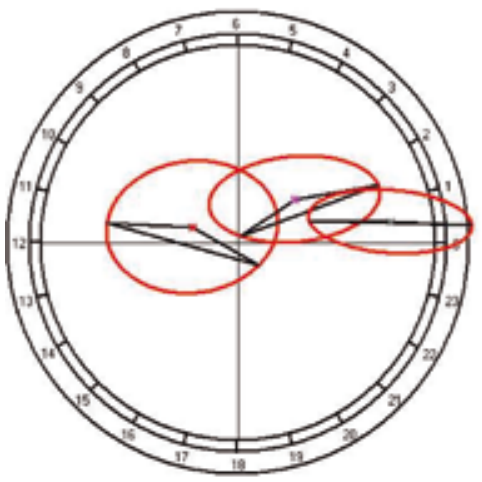

a

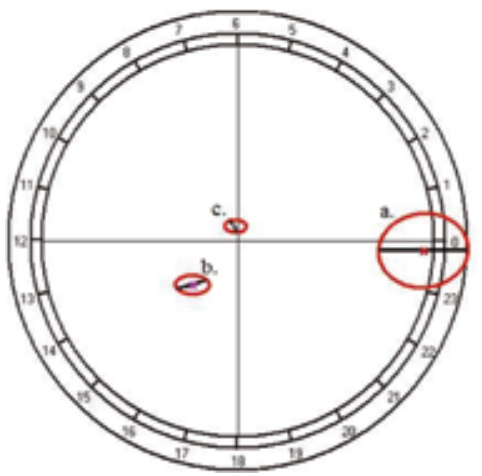

a

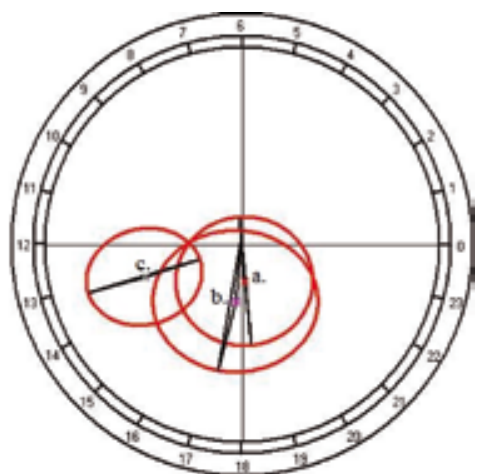

b

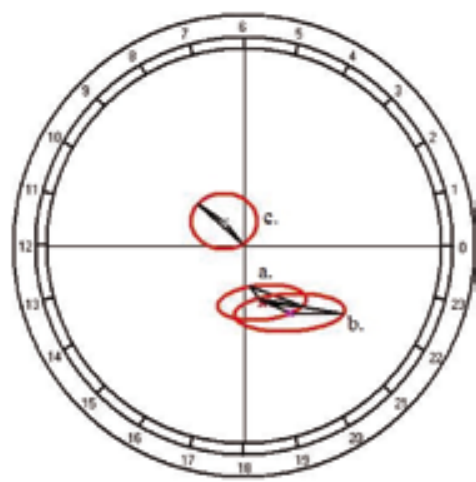

b

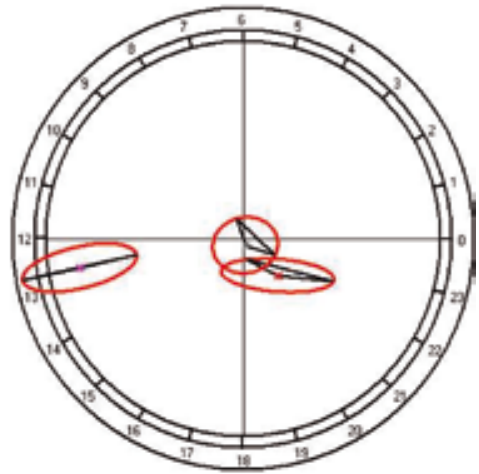

b

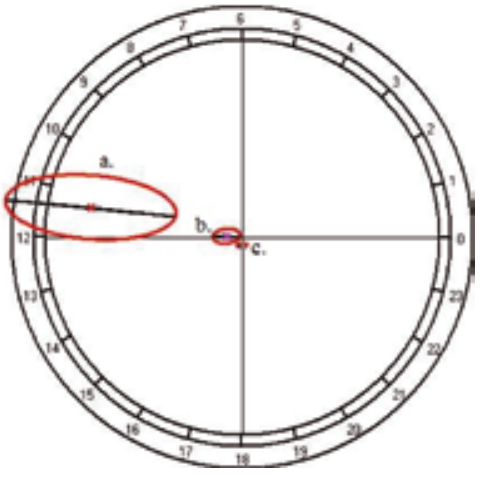

b
Fig. 1. Cosinor analysis of daily temperature dynamics at a fixed light regime (A) and under constant light conditions (B). Hereinafter:

$a$ - parameters of 3-month-old animals, $b$ - parameters of 6-month-old animals, $c$ parameters of 12-month-old animals

Fig. 2. Cosinor analysis of the daily dynamics of plasma glucose at a fixed light regime (A) and under constant light conditions (B)

Fig. 3. Cosinor analysis of the daily dynamics of total plasma protein at a fixed light regime (A) and under constant light conditions (B)

Fig. 4. Cosinor analysis of the daily dynamics of plasma albumin at a fixed light regime (A) and under constant light conditions (B) 
Halberg F. 2005. Chronoastrobiology: Vernadsky's future science. Мar. III Int. Conf.;24-4.

WeHr TA. 2001. Photoperiodism in humans and other primates: evidence and implications. J. Biol. Rhythms. 16(4):364-348. https://doi. org/10.1177/074873001129002060

Foster RG, Roenneberg T. 2008. Human responses to the geophysical daily, annual and lunar cycles. Current biology. 18(17):794-784. https://doi.org/10.1016/j. cub.2008.07.003

BOyCE PR. Human factors in lighting. Crc Press. 2014.

Tang C-HA, Hinteregger E, Shang Y, Rosbash M. 2010. Lightmediated TIM degradation within Drosophila pacemaker neurons ( $\mathrm{s}$-LNvs) is neither necessary nor sufficient for delay zone phase shifts. Neuron. 66(3):385-378. https://doi.org/10.1016/j. neuron.2010.04.015

SChmidt TM, Chen SK, Hattar S. 2011. Intrinsically photosensitive retinal ganglion cells: many subtypes, diverse functions. Trends Neurosci. 34(11):580-572. doi: 10.1016/j.tins.2011.07.001

Nasir-Ahmad S, Lee SC, Martin PR, Grünert U. 2019. Melanopsin-expressing ganglion cells in human retina: Morphology, distribution, and synaptic connections. Journal of Comparative Neurology. 527(1):327-312. https://doi.org/10.1002/cne.24176

RUSAK B. Photic enthronement routes in mammals. 1992: [Pap.] Meet. FESN Study Group «Circadian Rhythms». Discuss. Neurosci. 8(2-3): 43-38.

Harrington, ME. 1997. The ventral lateral geniculate nucleus and the intergeniculate leaflet: interrelated structures in the visual and circadian systems. Neurosci. Biobehav. Rev. 21(5):727-705. https://doi. org/10.1016/S0149-7634(96)00019-X

Ding G, Gong Y, Eckel-Mahan KL \& Sun Z. 2018. Central Circadian Clock Regulates Energy Metabolism. In Neural Regulation of Metabolism.103-79. Springer, Singapore. doi: 10.1007/978-981-13-12861 -5

Hastings, M. H., Maywood, E. S., Brancaccio, M. 2019. The Mammalian Circadian Timing System and the Suprachiasmatic Nucleus as Its Pacemaker. Biology, 8(1), 13. https://doi.org/10.3390/biology8010013

Martino, T. A., Young, M. E. 2015. Influence of the cardiomyocyte circadian clock on cardiac physiology and pathophysiology. Journal of biological rhythms, 30(3): 183-205. https://doi org/ $10.1177 / 0748730415575246$

Ogilvie K.M., Stetson M.H. 1997. The neuroendocrine control of clock-timed gonadotropin release in the female Syrian hamster: Role of serotonin. J. Endocrinol. 155(1):119-107. doi: 10.1677/joe.0.1550107

Chiesa, J. J., Duhart, J. M., Casiraghi, L. P., PaladiNo, N., Bussi, I. L., GolombeK, D. A. 2015. Effects of circadian disruption on physiology and pathology: From bench to clinic (and back). In Mechanisms of Circadian Systems in Animals and Their Clinical Relevance (pp. 289-320). Springer, Cham.
Moller M, Ravault JP, Cozzi B. 2009. The chemical neuroanatomy of the mammalian pineal-gland - neuropeptides. Neurochem. Int. 28(1): 33-23. https://doi. org/10.1016/0197-0186(95)00046-B

Iovino, M., Messana, T., De Pergola, G., Iovino, E., Guastamacchia, E., Giagulli, V. A., TrigGIANI, V. 2019. Vigilance states: central neural pathways, neurotransmitters and neurohormones. Endocrine, Metabolic \& Immune Disorders-Drug Targets (Formerly Current Drug Targets-Immune, Endocrine \& Metabolic Disorders), 19(1): 26-37. https://doi.org $/ 10.2174 / 1871530318666180816115720$

Robert, K. A., Lesku, J. A., Partecke, J., Chambers, B. 2015. Artificial light at night desynchronizes strictly seasonal reproduction in a wild mammal. Proceedings of the Royal Society B: Biological Sciences, 282(1816): 20151745. https://doi.org/10.1098/rspb.2015.1745

Touitou Y, SMolensky MH, Reinberg A. 2016. Factors that can alter the melatonin circadian rhythm. Chronobiology international. 33(9):1130-1129. http://dx.doi.org/10.1080/07420528.2016.1206911

Yurt, K. K., Kaplan, S., Kivrak, E. G. 2018. The neuroprotective effect of melatonin on the hippocampus exposed to diclofenac sodium during the prenatal period. Journal of chemical neuroanatomy, 87: 37-48. https://doi.org/10.1016/j.jchemneu.2017.05.006

Schwartz WJ, de la Iglesia HO, Zlomanczuk P, ILLNEROVA H. 2001. Encoding le quattrostagioni within the mammalian brain: photoperiodic orchestration through the suprachiasmatic nucleus. J. Biol. Rhythms. 16(4):311-302. https://doi.org/10.1177\% 2F074873001129002024

Arushanyan EB, Beier EV. 2001. The Hippocampus in the Time-course of Behaviour. Uspekhi Fiziol. Nauk. 32(1):95-79.

Serrano, J., Encinas, J. M., Fernandez, A. P., Rodrigo, J., Martinez, A. 2006. Effects of acute hypobaric hypoxia on the nitric oxide system of the rat cerebral cortex: Protective role of nitric oxide inhibitors. Neuroscience, 142(3): 799-808. https://doi. org/10.1016/j.neuroscience.2006.07.046

Ha M., Park J. 2005. Shiftwork and metabolic risk factors of cardiovascular disease. J. Occup. Health. 47: 95-89. https://doi.org/10.1539/joh.47.89

Jasser SA, Blask DE, Brainard GC. 2006. Light during darkness and cancer: relationships in circadian photoreception and tumor biology. Cancer Causes Control.17:523-515. doi:10.1539/joh.47.89

Knutsson A. Health disorders of shift workers. Occupat. Med. 2003; 53:108-103. https://doi.org/10.1093/oc$\mathrm{cmed} / \mathrm{kqg} 048$

Wang F., Zhang L., Zhang Y. 2014. Meta-analysis on night shift work and risk of metabolic syndrome. Obes. Rev.15:720-709. https://doi.org/10.1111/ obr. 12194

Fonken L.K., Workman J.L., Walton J.C. 2010. Light at night increases body mass by shifting the time of food intake. Proc. nat. Acad. Sci. USA. 107:18669- 
18664. https://doi.org/10.1073/pnas.1008734107

Vinogradova I, Anisimov V. 2013.Melatonin prevents the development of the metabolic syndrome in male rats exposed to different light/dark regimens. Biogerontology. 14:409-401. https://doi.org/10.1007/ s10522-013-9437-4

Gubin D, Cornelissen G, Halberg F. (1997). Halfweekly and weekly blood pressure patterns in late human ontogeny. Scripta med. 70: 216-207.

Gubin D, Gubin G. 1999. A circadian to extra-circadian variance transposition of human body temperature with advancing age. Chronobiol. Int.16:41.
Gubin D, Gubin G. 2001. Some general effects of aging upon circadian parameters of cardiovascular variables assessed longitudinally by ambulatory monitoring. Chronobiol. Int.18:1106.

Gubin DG, Gubin GD, Waterhouse J, Weinert D. 2006. The circadian body temperature rhythm in the elderly. Effect of single daily melatonin doses. Chronobiol. Int. 23: 658-639

Cornelissen G. 2014. Cosinor-based rhythmometry. Theoretical Biology and Medical Modelling. 11(1):16. https://doi.org/10.1186/1742-4682-11-16 